\title{
Redox status expressed as GSH:GSSG ratio as a marker for oxidative stress in paediatric tumour patients
}

\author{
ONDREJ ZITKA $^{1-3}$, SYLVIE SKALICKOVA ${ }^{1}$, JAROMIR GUMULEC ${ }^{1,4}$, MICHAL MASARIK $^{1,3,4}$, \\ VOJTECH ADAM ${ }^{1,3}$, JAROMIR HUBALEK $^{1-3}$, LIBUSE TRNKOVA $^{1,3,5}$, JARMILA KRUSEOVA $^{6}$, \\ TOMAS ECKSCHLAGER ${ }^{6}$ and RENE KIZEK ${ }^{1,3}$
}

\author{
${ }^{1}$ Department of Chemistry and Biochemistry, Faculty of Agronomy, Mendel University in Brno, Brno 61300; \\ ${ }^{2}$ Department of Microelectronics, Faculty of Electrical Engineering and Communication, Brno University of Technology, \\ Brno 61200; ${ }^{3}$ Central European Institute of Technology, Brno University of Technology, Brno 61600; \\ ${ }^{4}$ Department of Pathological Physiology, Faculty of Medicine, Masaryk University, Brno 62500; ${ }^{5}$ Department \\ of Chemistry, Faculty of Science, Masaryk University, Brno 62500; ${ }^{6}$ Department of Paediatric Hematology and \\ Oncology, 2nd Medical Faculty, Charles University and University Hospital Motol, Prague 15006, Czech Republic
}

Received June 11, 2012; Accepted August 29, 2012

DOI: $10.3892 / \mathrm{ol} .2012 .931$

\begin{abstract}
Oxidative stress causes profound alterations of various biological structures, including cellular membranes, lipids, proteins and nucleic acids, and it is involved in numerous malignancies. Reduced glutathione (GSH) is considered to be one of the most important scavengers of reactive oxygen species (ROS), and its ratio with oxidised glutathione (GSSG) may be used as a marker of oxidative stress. The main aim of this study was to determine GSH:GSSG ratio in the blood serum of paediatric cancer patients to use this ratio as a potential marker of oxidative stress. The whole procedure was optimised and the recoveries for both substances were greater than $80 \%$ under the optimised conditions. We analysed a group of paediatric patients $(n=116)$ with various types of cancer, including neuroblastoma, anaplastic ependymoma, germ cell tumour, genital tract tumour, lymphadenopathy, rhabdomyosarcoma, nephroblastoma, Ewing's sarcoma, osteosarcoma, Hodgkin's lymphoma, medulloblastoma and retinoblastoma. We simultaneously determined the levels of reduced and oxidised glutathione, and thus, its ratio in the blood serum of the patients. The highest ratio was observed in retinoblastoma patients and the lowest in anaplastic ependymoma. We were able to distinguish between the diagnoses based on the results of the obtained GSH:GSSG ratio.
\end{abstract}

Correspondence to: Professor Rene Kizek, Department of Chemistry and Biochemistry, Faculty of Agronomy, Mendel University, 1 Zemedelska, Brno 61300, Czech Republic

E-mail:kizek@sci.muni.cz

Key words: glutathione, paediatric tumour patients, oxidative stress, high performance liquid chromatography with electrochemical detection

\section{Introduction}

Reduced glutathione (GSH), a ubiquitous tripeptide thiol, is a vital intracellular and extracellular protective antioxidant, which plays a number of key and/or crucial roles in the control of signalling processes, detoxifying certain xenobiotics and heavy metals, as well as other functions. It is a tripeptide composed of cysteine, glutamic acid and glycine. Intracellular and whole blood concentrations of GSH are in the milimolar range, while the plasma concentration is in the micromolar range and accounts for approximately $0.4 \%$ of total blood GSH (1-5). The GSH synthesis and metabolism pathway is shown in Fig. 1. GSH is synthesised in the cell by $\gamma$-glutamylcysteine synthetase $(\gamma$-GCS) and glutathione synthetase (6). The $\gamma$-GCS-catalysed formation of $\gamma$-glutamylcysteine is the first and rate-limiting step in de novo GSH synthesis and is feedback-inhibited by GSH, a mechanism that is central to the regulation of cellular GSH concentrations (7). Thus, cysteine is a rate-limiting substrate for de novo GSH synthesis (8).

Within cells, total GSH exists free and bound to proteins. Since the enzyme glutathione reductase, which reverts free glutathione from its oxidised form (GSSG) is constitutively active and inducible upon oxidative stress, free glutathione exists almost exclusively in its reduced form. The ratio of reduced to oxidised glutathione within cells is often used as a marker of cellular toxicity (9-12). Under normal conditions, the GSH redox couple is well-known to be present in mammalian cells in the concentration range of 1-10 $\mathrm{mM}$. In a resting cell, the molar GSH:GSSG ratio exceeds 100:1, while in various models of oxidative stress, this ratio has been demonstrated to decrease to values of 10:1 and even 1:1 (13).

Oxidative stress is manifested by the excessive production of reactive oxygen species (ROS) in the face of insufficient or defective antioxidant defence systems. Oxidative stress causes profound alterations of various biological structures, including cellular membranes, lipids, proteins and nucleic acids. Oxidative stress is considered to be involved in ageing (14-20) 
and in various diseases, including diabetes mellitus (21-23), atherosclerosis (24,25), rheumatoid arthritis (26-29), Alzheimer's disease (30-32), Parkinson's disease (33-35) and cancer (36-44). There is an increasingly growing interest in identifying biomarkers for diseases, in which oxidative stress is involved (45).

For many years, GSH has been measured by several analytical methods. In particular, high performance liquid chromatography (HPLC) with various detection techniques including ultraviolet (UV) absorbance and fluorescence detection, mass spectrometry and/or electrochemical detection (ED) are commonly used for determination of GSH and GSSG concentrations (46-49). Each method has its advantages and limitations and may serve a particular need in analysis (50). $\mathrm{ED}$ is an attractive alternative method for electroactive species detection, due to its inherent advantages of simplicity, ease of miniaturisation, high sensitivity and relatively low cost. The aim of this study was to determine the GSH:GSSG ratio in the blood serum of paediatric cancer patients to use this ratio as a potential marker of oxidative stress. For determination of the GSH:GSSG ratio, HPLC-ED was optimised and used.

\section{Material and methods}

Chemicals and pH measurements. GSH, GSSG and trifluoroacetic acid (TFA) were purchased from Sigma-Aldrich (St. Louis, MO, USA). HPLC-grade methanol (>99.9\%; v/v) was obtained from Merck KGaA (Darmstadt, Germany). Other chemicals were purchased from Sigma-Aldrich unless otherwise stated. Stock standard solutions of the thiols $\left(1 \mathrm{mg} \cdot \mathrm{ml}^{-1}\right)$ were prepared with ACS water (Sigma-Aldrich) and stored at $-20^{\circ} \mathrm{C}$ in the dark. Working standard solutions were prepared daily by diluting the stock solutions. All solutions were filtered through $0.45-\mu \mathrm{m}$ nylon filter discs (Millipore, Billerica, MA, USA) prior to HPLC analysis. The $\mathrm{pH}$ value was measured using WTW inoLab Level 3 with terminal Level 3 (WTW $\mathrm{GmbH}$, Weilheim, Germany).

HPLC-ED analysis. The HPLC-ED system consists of two chromatographic pumps (Model 582; ESA, Inc., Chelmsford, MA, USA; working range $0.001-9.999 \mathrm{ml} / \mathrm{min}$ ), a chromatographic column with reverse phase Zorbax eclipse AAA C18 (Agilent Technologies, Inc., Santa Clara, CA, USA; $150 \times 4.6 \mathrm{~mm} ; 3.5-\mu \mathrm{m}$ particles) and a twelve-channel CoulArray electrochemical detector (Model 5600A; ESA, Inc.). The detector consists of three flow analytical chambers (Model 6210; ESA, Inc.). Each chamber contains four analytical cells and one analytical cell contains two referent (hydrogen-palladium), as well as two counters and porous graphite working electrodes. The ED is situated in the thermostated control module. A $20 \mu \mathrm{l}$ sample was injected using an autosampler (Model 542; ESA, Inc.), which has thermostated space for the column. The column was termostated at $35^{\circ} \mathrm{C}$. Other conditions were optimised and are described later.

Determination of recovery in real samples. Recovery of GSH and GSSG were evaluated with homogenates spiked with standards according to Causon (50). Prior to extraction, $100 \mu \mathrm{l}$ GSH and GSSG was added to the blood serum homogenate. Homogenates were blindly assayed and the concentration

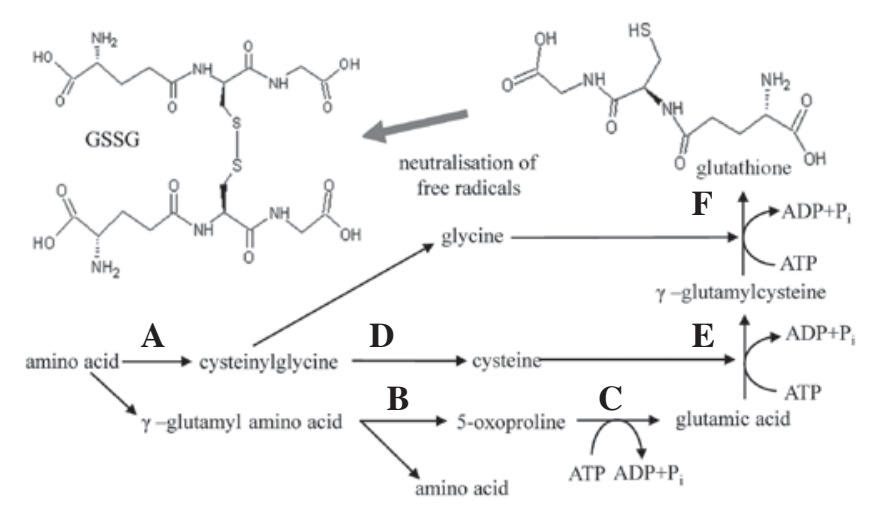

Figure 1. Scheme of $\gamma$-glutamyl cycle, the synthesis of GSH according particular steps. (A) $\gamma$-glutamyl transpeptidase, (B) $\gamma$-glutamyl cyclotransferase, (C) oxoprolinase, (D) peptidase, (E) $\gamma$-GCS, (F) glutathione synthetase and subsequent GSH scavenging of free radicals and self conversion to GSSG. GSSG, oxidised gluathione. GSH, reduced glutathione; $\gamma$-GCS, glutamyl-cysteine synthetase.

of GSH and GSSG was derived from the calibration curves. The spiking of GSH and GSSG was determined as a standard measured in the absence of real sample. Accuracy was evaluated by comparing the estimated concentration with the known concentrations of both thiols.

Human blood serum. Blood samples were obtained from 116 children hospitalised at the Department of Paediatric Haematology and Oncology (Faculty Hospital Motol, Prague, Czech Republic) with newly diagnosed solid tumours of neuroblastoma $(n=27)$, nephroblastoma $(n=8)$, anaplastic ependymoma $(n=4)$, Ewing's sarcoma $(n=9)$, germ cell tumour $(n=4)$, osteosarcoma $(n=16)$, tumour of the genital tract $(n=6)$, Hodgkin's lymphoma $(n=16)$, lymphadenopathy $(n=3)$, medulloblastoma $(n=15)$, rhabdomyosarcoma $(n=4)$ and retinoblastoma $(n=4)$. The study was approved by the ethics committee of Faculty Hospital Motol, Prague, Czech Republic. Written informed patient consent was obtained from the patients. Subjects ranged between 1 and 10 years of age. The blood samples were collected before chemotherapy and radiotherapy. Serum was separated by centrifugation at 4,000 x g for 10 min (Model 5402; Eppendorf AG, Hamburg, Germany), and the samples were stored at $-80^{\circ} \mathrm{C}$ until assayed. When required, the denatured samples were centrifuged at $15,000 \times \mathrm{g}$ at $4^{\circ} \mathrm{C}$ for $30 \mathrm{~min}$ (Model 5402; Eppendorf AG) and directly analysed using an optimised HPLC-ED method.

Descriptive statistics. Data were processed using Microsoft Excel (USA). Results are expressed as mean \pm standard deviation (SD) unless otherwise stated. The detection limits [3 signal/noise $(\mathrm{S} / \mathrm{N})$ ] were calculated according to Long and Winefordner (51), while $\mathrm{N}$ was expressed as the SD of noise determined in the signal domain unless otherwise noted.

\section{Results}

Optimisation of HPLC-ED method. Primarily, it was necessary to optimise the separation and subsequent ED in order to achieve the required accuracy and sensitivity for the determination of GSH and GSSG in real blood serum samples. 
A

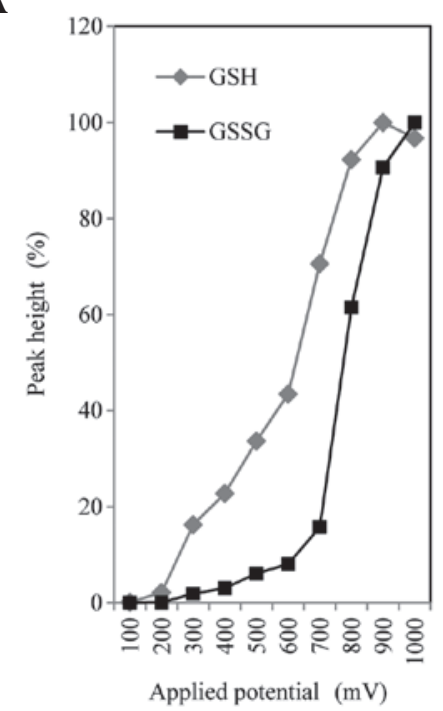

B

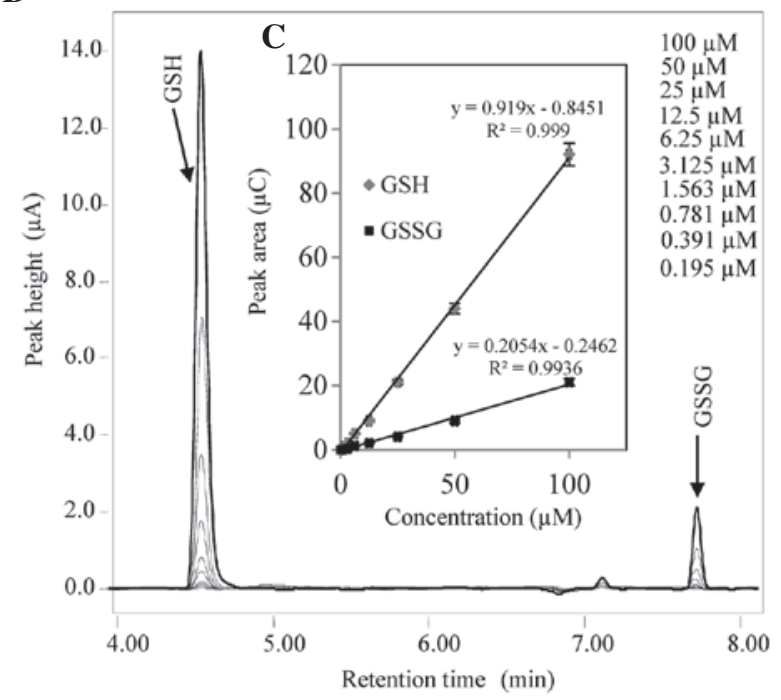

Figure 2. (A) HDV of GSH $(50 \mu \mathrm{M})$ and GSSG $(25 \mu \mathrm{M})$. (B) Overlay of typical HPL chromatograms of GSH and GSSG within the range of 0.2-100 $\mu \mathrm{M}$, and used for preparation of (C) calibration curves. Experimental conditions for the mobile phase were as follows: A, $80 \mathrm{mM}$ TFA; and B, $100 \%$ methanol. Compounds were eluted by following an increasing linear gradient: 0-1 min (3\% B), 1-2 min (10\% B), 2-5 min (30\% B) and 15-16 min (98\% B). Flow rate of mobile phase was $1 \mathrm{ml} / \mathrm{min}$, and an electrode potential of $900 \mathrm{mV}$ was used. GSH, reduced glutathione, GSSG, oxidised gluathione. HDV, hydrodynamic voltammogram; HPL, high performance liquid; TFA, trifluoroacetic acid.

Therefore, we focused on studying the influence of flow rate, concentration of components of the mobile phase, elution and applied potential of the working electrodes on GSH and GSSG signals.

Flow rate. The mobile phase flow rate is an important parameter influencing the electrochemical response of the detector. When using a chromatographic column Zorbax Eclipse AAA, optimum mobile phase flow rate was $1 \mathrm{ml} / \mathrm{min}$ at pressures of 130 bars. Additionally, we identified that if the flow $>1 \mathrm{ml} / \mathrm{min}$, the responses of GSH and GSSG decreased by $>10 \%$. This is probably caused by reducing the time-concentration of the analyte on the electrode surface. Even with a lower flow rate, a decreased signal occurred compared with the maximum, but the total peak area remained the same with a tolerance of $7 \%$. Although a lower flow rate may not be significantly affected by resolution, it may extend the period of separation, which is critical for analysing a large number of clinical samples. Therefore, we decided to use $1 \mathrm{ml} / \mathrm{min}$ as the optimum flow rate of the mobile phase.

Influence of methanol on ED. Achieving an optimal resolution is crucial for simultaneous separation of analytes. In order to separate all determined substances in the system with reversed-phase, a gradient with the increasing content of organic solvent is required. Since the electrochemical determination of substances contained in the sample requires the presence of an electrolyte, we examined the effect of the organic solvent (methanol) on the electrochemical response of analytes. We identified that $15 \%$ content of methanol in the mobile phase, which is the polar component of the mobile phase composed also from $80 \mathrm{mM}$ TFA, lead to more than $50 \%$ decrease in GSH signal. A marked decline of GSSG signal was also observed. The best ratio of $80 \mathrm{mM}$ TFA and methanol in the mobile phase was 99:1 (v/v).
Optimisation of gradient. If GSH and GSSG were separated by isocratic elution where the ratio of TFA and methanol was 99:1 (v/v), it would be the most sensitive, but the retention times of the separated substances would be too high. A significant tailing of peaks was observed during the elution of compounds with higher retention under these conditions. Therefore, we optimised the increasing content of methanol with respect to the sensitivity of the analysis. Based on the optimisation steps, the mobile phase, which consisted of (A) $80 \mathrm{mM}$ TFA and (B) $100 \%$ methanol, was used for separation and detection of GSH and GSSG. Compounds were eluted by following an increasing linear gradient: $0-1 \mathrm{~min}(3 \% \mathrm{~B})$, $1-2 \min (10 \% \mathrm{~B}), 2-5 \mathrm{~min}(30 \% \mathrm{~B})$ and $15-16 \min (98 \% \mathrm{~B})$. Flow rate of the mobile phase was $1 \mathrm{ml} / \mathrm{min}$, and the time of one analysis inducing column regeneration was $20 \mathrm{~min}$.

$E D$. Sensitivity of the electrochemical detector may be more influenced by factors including the type of electrolyte in the mobile phase, concentration, $\mathrm{pH}$ and, in particular, applied potential. TFA was used as an ion-pair reagent, which provides the best separation conditions in the parameters mentioned above, and at a concentration of $80 \mathrm{mM}$ it is also an extremely suitable electrolyte for the detection of thiols. We further studied the effect of the applied potential on the working electrode set separately for GSH and GSSG, which were designed for hydrodynamic voltammogram (HDV). Tested potentials were 100, 200, 300, 400, 500, 600, 700, 800,900 and $1,000 \mathrm{mV}$. The responses detected at $100 \mathrm{mV}$ were negligible; however, when the potential reached $300 \mathrm{mV}$, detectable signals for GSH and GSSG were observed. While the GSH signal markedly increased from $600 \mathrm{mV}$, the GSSG signal markedly increased from $700 \mathrm{mV}$. This is probably due to the requirement for greater power for partial dissociation of the -S-S-group on the surface of the working electrode, in comparison to the relatively easily accessible $-\mathrm{SH}$ moiety of 


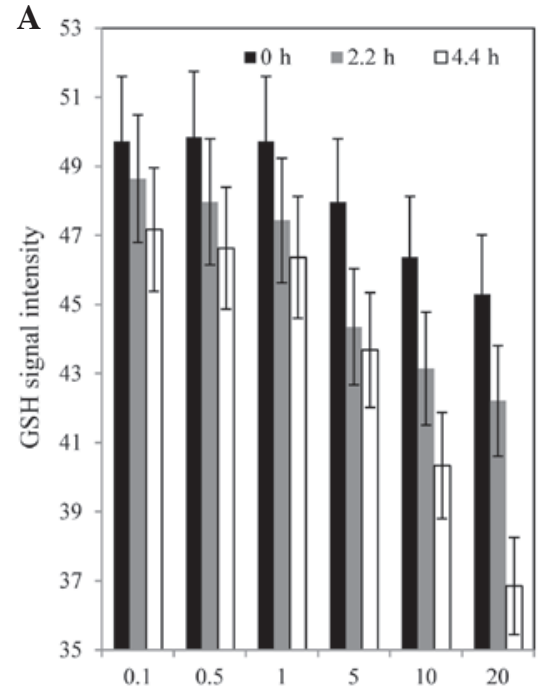

Applied concentration of phosphate buffer $(\mathrm{mM})$

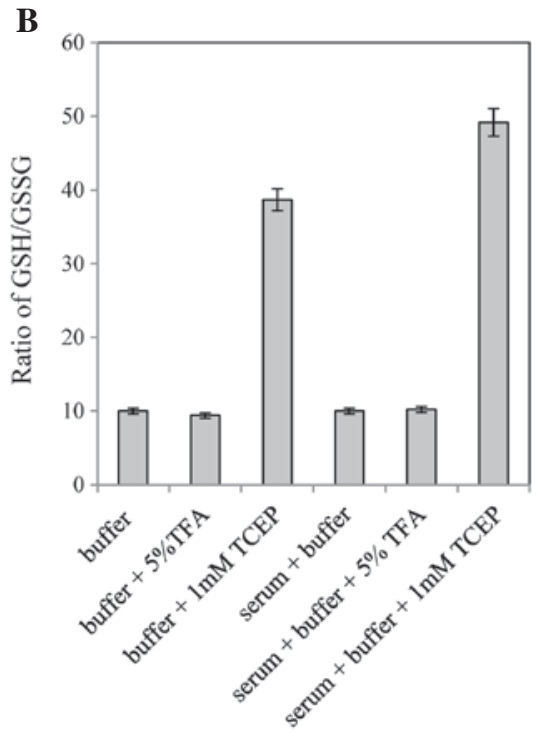

Figure 3. (A) Influence of phosphate buffer under various applied concentrations on GSH (50 $\mu \mathrm{M})$ isolation. (B) Influence of 5\% TFA and $1 \mathrm{mM}$ TCEP on GSH:GSSG ratio. The same concentration of GSH $(50 \mu \mathrm{g} / \mathrm{ml})$ and GSSG $(5 \mu \mathrm{g} / \mathrm{ml})$ was used. GSH, reduced glutathione, GSSG, oxidised gluathione; TFA, trifluoroacetic acid; TCEP, tris(2-carboxylethyl)phosphine.

GSH. We observed the highest signals for both compounds when a potential of $900-1,000 \mathrm{mV}$ was applied, which is evident from the HDVs showed in Fig. 2A. Based on the HDV results we were able to evaluate that the best glutathione detection was achieved when a potential of $900 \mathrm{mV}$ was applied to the working electrodes.

Calibration parameters. After identifying the optimal separation and detection conditions, the calibration curves for GSH and GSSG were measured within the concentration range of 0.2-100 $\mu \mathrm{M}$. Overlay of HPL chromatograms is shown in Fig. 2B, and the calibration curves are shown in Fig. 2C. The obtained dependences were strictly linear with $\mathrm{R}^{2}=0.9997$ for GSH and $\mathrm{R}^{2}=0.9936$ for GSSG. Detection limits $(3 \mathrm{~S} / \mathrm{N})$ were estimated with nanomolar subunits for both substances of interest.

Sample pretreatment for GSH:GSSG ratio determination. Prior to chromatographic analysis, precipitation of proteins with TFA to avoid excessive clogging of filters and precolumns, which protect the separation column from contaminations, was required. The proteins may interfere with detected substances and the obtained chromatograms may be extremely difficult to analyse. The denatured sample was than centrifuged and the resulting supernatant was directly injected to the chromatographic column. To ensure the lowest possible loss of target compounds during sample preparation it was necessary to examine several factors of a sample treatment, which could affect the overall recovery of GSH and GSSG.

Stability of GSH. Given that the formation of complexes may be faster under certain conditions ( $\mathrm{pH}$ and ionic strength), we decided to investigate the possibility of GSH complex formation in the solution used for isolation. The formation of the complex was determined via a decrease in the GSH peak. Primarily, we examined the effect of molar concentrations of phosphate buffer $(0.1,0.5,1,5,10$ and $20 \mathrm{mM}$; pH 7.5) on the GSH $(50 \mu \mathrm{g} / \mathrm{ml})$ signal. These samples were left following preparation at room temperature, and were analysed by HPLC at time $0,130(2.2 \mathrm{~h})$ and $260 \mathrm{~min}(4.3 \mathrm{~h})$. Based on the results obtained, higher concentrations of buffer caused a decreasing GSH signal, i.e. concentration; thus, $20 \mathrm{mM}$ phosphate buffer caused the highest decrease of glutathione concentration. It is clear that the greatest stability of GSH was observed in samples prepared in the presence of low concentrations of phosphate buffer.

Specifically, the lowest loss of glutathione occurred at the applied concentrations of 0.1-1 mM (Fig. 3A). These results clearly demonstrated that lower concentrations of phosphate buffer contribute to the stability of the sample. Therefore, for further experiments we used $1 \mathrm{mM}$ phosphate buffer ( $\mathrm{pH}$ 7.5).

Influence of various chemicals on GSH:GSSG ratio. To determine the extent of oxidative stress by glutathione it is necessary to know the ratio of GSH:GSSG. Therefore, we were aimed to determine whether TFA, which is normally added to the sample due to deproteination, could have an effect on GSSG level. We also studied the effect of adding the reducing agent tris(2-carboxylethyl)phosphine (TCEP), which may markedly influence the ratio of GSH:GSSG. Studies on TFA and TCEP were conducted in buffer and blood sera, and all variants were prepared with the same concentration of $50 \mu \mathrm{g} / \mathrm{ml} \mathrm{GSH}$ and $5 \mu \mathrm{g} / \mathrm{ml} \mathrm{GSSG}$. Samples were prepared in the presence of (i) $1 \mathrm{mM}$ phosphate buffer ( $\mathrm{pH} 7.5$ ), (ii) $1 \mathrm{mM}$ phosphate buffer ( $\mathrm{pH} 7.5$ ) with $5 \%$ TFA (v/v), and (iii) $1 \mathrm{mM}$ phosphate buffer ( $\mathrm{pH} 7.5$ ) with $1 \mathrm{mM}$ TCEP. To be able to assess the influence of the matrix, samples of blood serum were prepared in the same way. All samples were vortexed for 1 min and immediately analysed by HPLC following preparation. The GSH:GSSG ratio was determined, where the ratio of 10 was taken as a control. In the case of using 5\% TFA, $\pm 7 \%$ change from control was determined in variants of buffer and serum (Fig. 3B). The results reveal that TFA did not affect the ratio of GSH:GSSG. However, following the addition of 
Table I. Recovery of GSH and GSSG for blood serum sample analysis $(n=5)$.

\begin{tabular}{lcccc}
\hline Substance of interest & Homogenate $(\mu \mathrm{g} / \mathrm{ml})$ & Spiking $(\mu \mathrm{g} / \mathrm{ml})$ & Homogenate + spiking $(\mu \mathrm{g} / \mathrm{ml})$ & Recovery $(\%)$ \\
\hline GSH & $54 \pm 6$ & $50 \pm 5$ & $86 \pm 10$ & 83 \\
GSSG & $25 \pm 4$ & $10 \pm 2$ & $31 \pm 3$ & 89 \\
\hline
\end{tabular}

GSH, reduced gluthione; GSSG, oxidised gluthione.
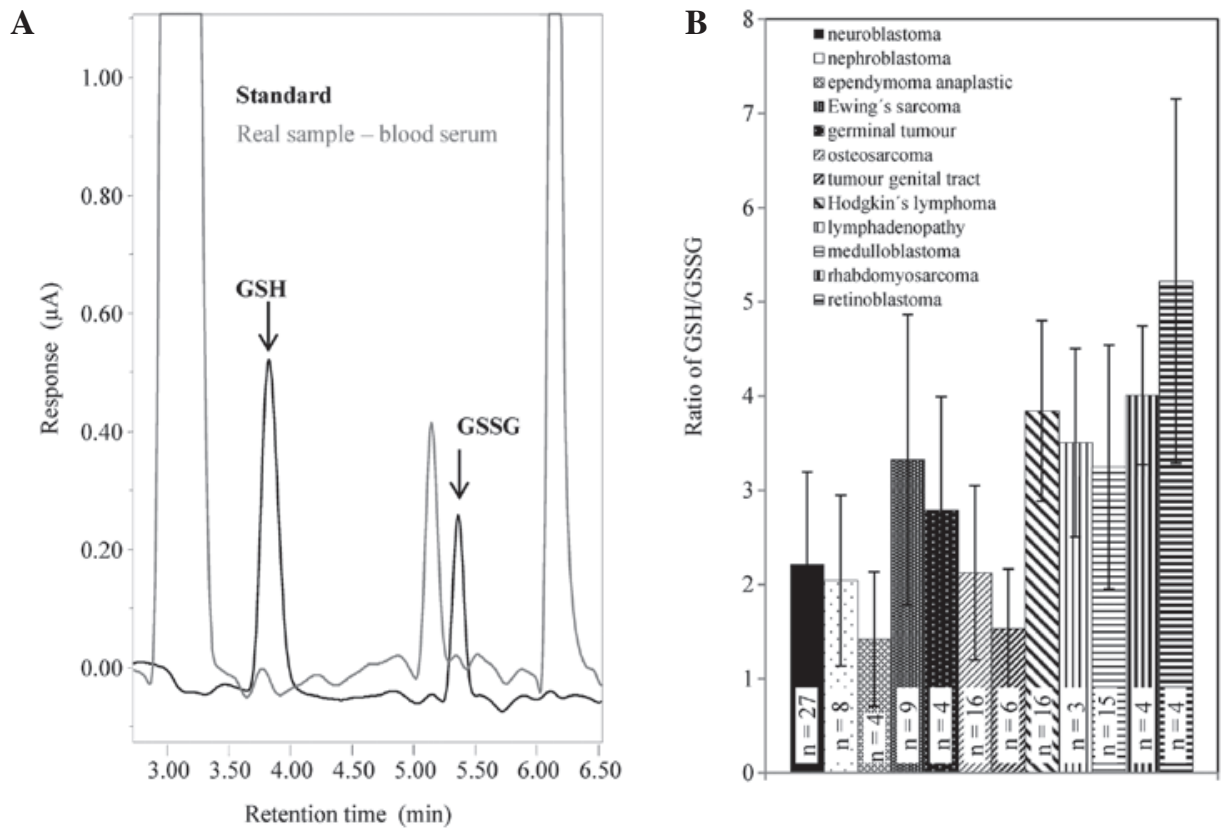

Figure 4. (A) Overlay of HPL chromatograms of a standard mixture of GSH (50 $\mu \mathrm{M})$ and GSSG $(25 \mu \mathrm{M})$ and real blood serum samples. (B) Ratio of GSH:GSSG determined in patients suffering from: neuroblastoma $(n=27, R S D=43.9 \%)$, nephroblastoma $(n=8, R S D=44.3 \%)$, anaplastic epemdymoma $(n=4, R S D=50.3 \%)$, Ewing's sarcoma ( $n=9, \mathrm{RSD}=46.4 \%)$, germ cell tumour $(\mathrm{n}=4, \mathrm{RSD}=43.1 \%)$, osteosarcoma $(\mathrm{n}=16, \mathrm{RSD}=43.6 \%)$, tumour of the genital tract $(\mathrm{n}=6$, $\mathrm{RSD}=41.5 \%)$, Hodgkin's lymphoma ( $\mathrm{n}=16, \mathrm{RSD}=24.9 \%)$, lymphadenopathy $(\mathrm{n}=3, \mathrm{RSD}=22.1 \%)$, medulloblastoma $(\mathrm{n}=15, \mathrm{RSD}=39.8 \%)$, rhabdomyosarcoma $(\mathrm{n}=4, \mathrm{RSD}=18.4 \%)$ or retinoblastoma $(n=4, \mathrm{RSD}=37.0 \%)$. GSH, reduced glutathione, GSSG, oxidised gluathione. HPL, high performance liquid; RSD, relative standard deviation.

TCEP, there was a significant increase in the ratio to 38 and 48 in the buffer and blood serum, respectively. TCEP reduced the majority of GSSG to GSH, which was the reason for the significant increase of the GSH:GSSG ratio. In the case of blood serum, the ratio was even higher compared with buffer. This phenomenon may be explained by the involvement of the biological matrix in a non-specific reaction of the complexes or the presence of certain concentrations of glutathione bound to the matrix constituents. These results clearly indicate that TCEP reduces GSSG back to GSH, which could be used to determine the total amount of glutathione.

Recovery of pretreatment. Recovery estimation for sample preparation and analysis for a sample of blood serum using an optimised separation method was conducted by adding $10 \mu \mathrm{g}$ / $\mathrm{ml} \mathrm{GSH}$ and $10 \mu \mathrm{g} / \mathrm{ml} \mathrm{GSSG}$ prior to precipitation with 5\% TFA and subsequent centrifugation. A sample with a GSH:GSSG ratio of 2.8 was used for determining recovery. The resulting recoveries are indicated in Table I. A recovery estimation of 83 and $89 \%$ for GSH and GSSG, respectively, clearly follows from the results previously obtained. GSH recovery can be associated with the imperfect protection of free-SH groups of glutathione, which can interact with the remains of biological matrices, and thus reduce the total concentration of free GSH during the preparation of the samples.

Determination of GSH:GSSG ratio in paediatric patients. The antioxidant function of GSH is primarily due to its involvement in enzymatic pathways that cells have developed against ROS. The most important pathway involves glutathione peroxidase (GPx) and glutathione reductase (GR). GPx catalyses the reduction of hydrogen peroxide, which is produced by superoxide dismutase (SOD) through the dismutation of superoxide anions or organic hydroperoxides. GSH and GSH-dependent enzymes act in cooperation to scavenge ROS and/or neutralise their toxic oxidising effect. These systems act at the same time and in cooperation to protect the human body from ROS. Under oxidative stress conditions, GSH is oxidised to GSSG; thus, the GSH:GSSG ratio is altered.

\section{Discussion}

The GSH:GSSG ratio may be used as a marker of oxidative stress, which arises due to various malignancies. Using the 
optimised method, we were able to analyse real samples of paediatric patients (Fig. 4A). GSH and GSSG concentrations identified in each sample were recalculated to recovery, and based on these values, the GSH:GSSG ratios were given. The lowest number of patients in a group $(n=3)$ were diagnosed with lymphadenopathy and the highest number $(n=27)$ were diagnosed with neuroblastoma. Average values of GSH:GSSG ratio are demonstrated in Fig. 4B. The results reveal that the lowest redox status, which is given by the GSH:GSSG ratio of 1.4, was identified in patients diagnosed with ependymoma anaplastic, and the second lowest ratio of 1.5 was identified in patients diagnosed with genital tract tumour. The average values of both groups of patients also had a large relative standard deviation (RSD) of 50.3 and $41.5 \%$, respectively. The lowest RSDs were identified in lymphadenopathy and rhabdomyosarcoma patients with a higher GSH:GSSG ratio of 4.0 and 3.5, where RSDs were 18.4 and 22.1, respectively. Additionally, the lowest oxidative damage, expressed as a GSH:GSSG ratio of 5.2, was revealed in retinoblastoma patients.

\section{Acknowledgements}

Financial support from NANOSEMED GA AV (Grant No. KAN208130801), NanoBioTECell GA CR (Grant No. 102/11/1068), CEITEC (Grant No. CZ.1.05/1.1.00/02.0068) and and the Project for Conceptual Development of Research Organization (00064203) is gratefully acknowledged.

\section{References}

1. Lochman P, Adam T, Friedecky D, Hlidkova E and Skopkova Z: High-throughput capillary electrophoretic method for determination of total aminothiols in plasma and urine. Electrophoresis 24: 1200-1207, 2003.

2. Michelet F, Gueguen R, Leroy P, Wellman M, Nicolas A and Siest G: Blood and plasma glutathione measured in healthy-subjects by HPLC: relation to sex, aging, biological variables, and life habits. Clin Chem 41: 1509-1517, 1995.

3. Pastore A, Massoud R, Motti C, et al: Fully automated assay for total homocysteine, cysteine, cysteinylglycine, glutathione, cysteamine, and 2-mercaptopropionylglycine in plasma and urine. Clin Chem 44: 825-832, 1998.

4. Richie JP, Skowronski L, Abraham P and Leutzinger Y: Blood glutathione concentrations in a large-scale human study. Clin Chem 42: 64-70, 1996.

5. Shea M and Howell S: High-performance liquid chromatographic measurement of exogenous thiosulfate in urine and plasma. Anal Biochem 140: 589-594, 1984

6. Meister A and Tate SS: Glutathione and related gamma-glutamyl compounds: biosynthesis and utilization. Annu Rev Biochem 45: $559-604,1976$

7. Griffith OW and Meister A: Potent and specific-inhibition of glutathione synthesis by buthionine sulfoximine (S-n-butyl homocysteine sulfoximine). J Biol Chem 254: 7558-7560, 1979.

8. Meister A, Anderson ME and Hwang O: Intracellular cysteine and glutathione delivery systems. J Am Coll Nutr 5: 137-151, 1986.

9. Carelli S, Ceriotti A, Cabibbo A, Fassina G, Ruvo M and Sitia R: Cysteine and glutathione secretion in response to protein disulfide bond formation in the ER. Science 277: 1681-1684, 1997.

10. Locigno R and Castronovo V: Reduced glutathione system: role in cancer development, prevention and treatment (review). Int J Oncol 19: 221-236, 2001.

11. Noctor G and Foyer $\mathrm{CH}$ : Ascorbate and glutathione: keeping active oxygen under control. Annu Rev Plant Physiol Mol Biol 49: 249-279, 1998

12. Townsend DM, Tew KD and Tapiero H: The importance of glutathione in human disease. Biomed Pharmacother 57: 145-155, 2003.
13. Chai YC, Ashraf SS, Rokutan K, Johnston RB Jr and Thomas JA: S-thiolation of individual human neutrophil proteins including actin by stimulation of the respiratory burst: evidence against a role for glutathione disulfide. Arch Biochem Biophys 310: 273-281, 1994.

14. Arranz L, Fernández C, Rodríguez A, Ribera JM and De la Fuente M: The glutathione precursor $\mathrm{N}$-acetylcysteine improves immune function in postmenopausal women. Free Radic Biol Med 45: 1252-1262, 2008

15. Hashimoto K, Takasaki W, Yamoto T, Manabe S, Sato I and Tsuda S: Effect of glutathione (GSH) depletion on DNA damage and blood chemistry in aged and young rats. J Toxicol Sci 33: 421-429, 2008

16. Christon R, Haloui RB and Durand G: Dietary polyunsaturated fatty acids and aging modulate glutathione-related antioxidants in rat liver. J Nutr 125: 3062-3070, 1995.

17. Maher P: The effects of stress and aging on glutathione metabolism. Ageing Res Rev 4: 288-314, 2005.

18. Rebrin I, Bayne AC, Mockett RJ, Orr WC and Sohal RS: Free aminothiols, glutathione redox state and protein mixed disulphides in aging Drosophila melanogaster. Biochem J 382: 131-136, 2004.

19. Rebrin I and Sohal RS: Pro-oxidant shift in glutathione redox state during aging. Adv Drug Deliv Rev 60: 1545-1552, 2008.

20. Samiec PS, Drews-Botsch C, Flagg EW, et al: Glutathione in human plasma: decline in association with aging, age-related macular degeneration, and diabetes. Free Radic Biol Med 24: 699-704, 1998.

21. Cerielo A, Motz E, Cavarape A, et al: Hyperglycemia counterbalances the antihypertensive effect of glutathione in diabetic patients: evidence linking hypertension and glycemia through the oxidative stress in diabetes mellitus. J Diabetes Complications 11: 250-255, 1997.

22. Dincer Y, Akcay T, Alademir Z and Ilkova H: Effect of oxidative stress on glutathione pathway in red blood cells from patients with insulin-dependent diabetes mellitus. Metabolism 51: 1360-1362, 2002.

23. Yoshida K, Hirokawa J, Tagami S, Kawakami Y, Urata Y and Kondo T: Weakened cellular scavenging activity against oxidative stress in diabetes mellitus: regulation of glutathione synthesis and efflux. Diabetologia 38: 201-210, 1995.

24. Margutti P, Matarrese P, Conti F, et al: Autoantibodies to the C-terminal subunit of RLIP76 induce oxidative stress and endothelial cell apoptosis in immune-mediated vascular diseases and atherosclerosis. Blood 111: 4559-4570, 2008.

25. Signorelli SS, Neri S, Di Pino L, et al: Oxidative stress and endothelial damage in patients with asymptomatic carotid atherosclerosis. Clin Exp Med 1: 9-12, 2001.

26. Hassan MQ, Hadi RA, Al-Rawi ZS, Padron VA and Stohs SJ: The glutathione defense system in the pathogenesis of rheumatoid arthritis. J Appl Toxicol 21: 69-73, 2001.

27. Pedersen-Lane JH, Zurier RB and Lawrence DA: Analysis of the thiol status of peripheral blood leukocytes in rheumatoid arthritis patients. J Leukoc Biol 81: 934-941, 2007.

28. Seven A, Guzel S, Aslan M and Hamuryudan V: Lipid, protein, DNA oxidation and antioxidant status in rheumatoid arthritis. Clin Biochem 41: 538-543, 2008.

29. Karelson E, Mahlapuu R, Zilmer M, Soomets U, Bogdanovic N and Langel $\mathrm{U}$ : Possible signaling by glutathione and its novel analogue through potent stimulation of frontocortical $\mathrm{G}$ proteins in normal aging and in Alzheimer's disease. In: Cell Signaling, Transcription, and Translation as Therapeutic Targets. Vol 973. Diederich M (ed). New York Academy of Sciences, New York, pp537-540, 2002.

30. Liu HL, Wang H, Shenvi S, Hagen TM and Liu RM: Glutathione metabolism during aging and in Alzheimer disease. In: Strategies for Engineered Negligible Senescence: Why Genuine Control of Aging May Be Foreseeable. Vol 1019. De Grey ADN (ed). New York Academy of Sciences, New York, pp346-349, 2004.

31. Resende R, Moreira PI, Proenca T, et al: Brain oxidative stress in a triple-transgenic mouse model of Alzheimer disease. Free Radic Biol Med 44: 2051-2057, 2008.

32. Lang AE: The progression of Parkinson disease: a hypothesis. Neurology 68: 948-952, 2007.

33. Spina MB and Cohen G: Dopamine turnover and glutathione oxidation: implications for Parkinson disease. Proc Natl Acad Sci USA 86: 1398-1400, 1989.

34. Yamamoto N, Sawada H, Izumi Y, et al: Proteasome inhibition induces glutathione synthesis and protects cells from oxidative stress: relevance to Parkinson disease. J Biol Chem 282: 4364-4372, 2007. 
35. Barranco SC, Perry RR, Durm ME, et al: Relationship between colorectal cancer glutathione levels and patient survival: early results. Dis Colon Rectum 43: 1133-1140, 2000.

36. Kigawa J, Minagawa Y, Kanamori Y, et al: Glutathione concentration may be a useful predictor of response to second-line chemotherapy in patients with ovarian cancer. Cancer 82: $697-702,1998$.

37. Kumar A, Sharma S, Pundir CS and Sharma A: Decreased plasma glutathione in cancer of the uterine cervix. Cancer Lett 94: 107-111, 1995.

38. Wong DYK, Hsiao YL, Poon CK, et al: Glutathione concentration in oral cancer tissues. Cancer Lett 81: 111-116, 1994.

39. Yeh CC, Hou MF, Wu SH, et al: A study of glutathione status in the blood and tissues of patients with breast cancer. Cell Biochem Funct 24: 555-559, 2006.

40. Droge W: Free radicals in the physiological control of cell function. Physiol Rev 82: 47-95, 2002.

41. Hayes JD and Pulford DJ: The glutathione S-Transferase supergene family: regulation of GST and the contribution of the isoenzymes to cancer chemoprotection and drug resistance. Crit Rev Biochem Mol Biol 30: 445-600, 1995.

42. Valko M, Rhodes CJ, Moncol J, Izakovic M and Mazur M: Free radicals, metals and antioxidants in oxidative stress-induced cancer. Chem Biol Interact 160: 1-40, 2006.

43. Hayes JD and McLellan LI: Glutathione and glutathione-dependent enzymes represent a co-ordinately regulated defence against oxidative stress. Free Radic Res 31: 273-300, 1999.
44. Dalle-Donne I, Rossi R, Colombo R, Giustarini D and Milzani A: Biomarkers of oxidative damage in human disease. Clin Chem 52: 601-623, 2006

45. Klejdus B, Zehnálek J, Adam V, et al: Sub-picomole high-performance liquid chromatographic/mass spectrometric determination of glutathione in the maize (Zea mays L.) kernels exposed to cadmium. Anal Chim Acta 520: 117-124, 2004.

46. Potesil D, Petrlova J, Adam V, et al: Simultaneous femtomole determination of cysteine, reduced and oxidized glutathione, and phytochelatin in maize (Zea mays L.) kernels using high-performance liquid chromatography with electrochemical detection. J Chromatogr A 1084: 134-144, 2005.

47. Petrlova J, Mikelova R, Stejskal K, et al: Simultaneous determination of eight biologically active thiol compounds using gradient elution-liquid chromatography with Coul-Array detection. J Sep Sci 29: 1166-1173, 2006.

48. Zitka O, Huska D, Krizkova S, et al: An investigation of glutathione-platinum(II) interactions by means of the flow injection analysis using glassy carbon electrode. Sensors 7: 1256-1270, 2007.

49. Iwasaki Y, Saito Y, Nakano Y, et al: Chromatographic and mass spectrometric analysis of glutathione in biological samples. J Chromatogr B Analyt Biomed Life Sci 877: 3309-3317, 2009.

50. Causon R: Validation of chromatographic methods in biomedical analysis - viewpoint and discussion. J Chromatogr B 689: 175-180, 1997. 